\title{
A New Model to Predict Productivity of Multiple-Fractured Horizontal Well in Naturally Fractured Reservoirs
}

\author{
Junchao Wang, Haitao Li, Yongqing Wang, Ying Li, Beibei Jiang, and Wei Luo \\ State Key Laboratory of Oil and Gas Reservoir Geology and Exploitation, Southwest Petroleum University, Chengdu 610500, China \\ Correspondence should be addressed to Junchao Wang; wangjunchao2012@126.com
}

Received 6 October 2014; Revised 17 January 2015; Accepted 17 January 2015

Academic Editor: Hak-Keung Lam

Copyright (C) 2015 Junchao Wang et al. This is an open access article distributed under the Creative Commons Attribution License, which permits unrestricted use, distribution, and reproduction in any medium, provided the original work is properly cited.

\begin{abstract}
In order to predict productivity of multiple-fractured horizontal well in fractured reservoir, flow models of reservoir and hydraulic fractures based on the volumetric source idealization are developed. The models are solved by utilizing Laplace transformation and orthogonal transformation, and flow rate of the well is calculated by coupling the two models. Compared to traditional point source functions, volumetric source function has many advantages in properties of function and programming calculation. The productivity predicting model is verified via an analytical ternary-porosity model. Moreover, a practical example of fractured horizontal well is studied to analyze the productivity and its influent factors. The result shows that flow rate of each fracture is different and inner fracture contributes least to productivity. Meanwhile, there are optimizing ranges for number, length, and conductivity of hydraulic fractures. In low-permeability reservoir, increasing surface area in contact with reservoir by increasing number and length of hydraulic fractures is the most effective method to improve the productivity.
\end{abstract}

\section{Introduction}

Hydraulic fracturing is widely applied to improve the productivity of horizontal wells. Analytical methods to predict the productivity of fractured horizontal well have become invalid tools to optimize the hydraulic fracturing and determine productivity of horizontal well. There are two main methods to accomplish the target, which are analytical flow models [16] and source function (Green function) models [7-16].

Analytical flow models such as triple-linear model [1, 2] and ternary-porosity model [3] were built to simulate productivity of fractured horizontal well. But these models are limited in application $[17,18]$; the triple-linear model is not accurate if the regions beyond the well tips control the well performance; the triporosity model could not simulate the properties of natural fractures very well.

In order to obtain more practical solutions, point source functions [19-21] for fractured reservoirs are developed to simulate pressure behavior and productivity. Application of source functions (Green's functions) is the main technique since Gringarten and Ramey Jr. [22] introduced methods to solve transient flow problems for the first time. They also studied the application of Newman's principle to transform 3dimensional system into the product of three 1-dimensional systems. As for simplification, subsequent works have been focusing on point source functions which neglected the flow in source and its volume. Ogunsanya et al. [23, 24] pointed out that point source functions have computational problems and the no volume assumption may not be adequate in some specific situations, and then they revisited the concept of volumetric source and presented a new "solid bar source" model. Amini et al. [11-14] developed distributed volumetric source (DVS) model of homogeneous reservoirs and validated the applications in vertical well, horizontal well, and fractured vertical well with well-known existing solutions. Amini and Valkó [14] also demonstrated the application of DVS model in fractured horizontal well and emphasized advantages of the method for volumetric source. The solution of volumetric source model does not suffer from the problem of inherent singularity, and pressure can be calculated at any point. Meanwhile, it is more adjustable than point source solutions; the solution is always in the same form, no matter what the types of the source are. 


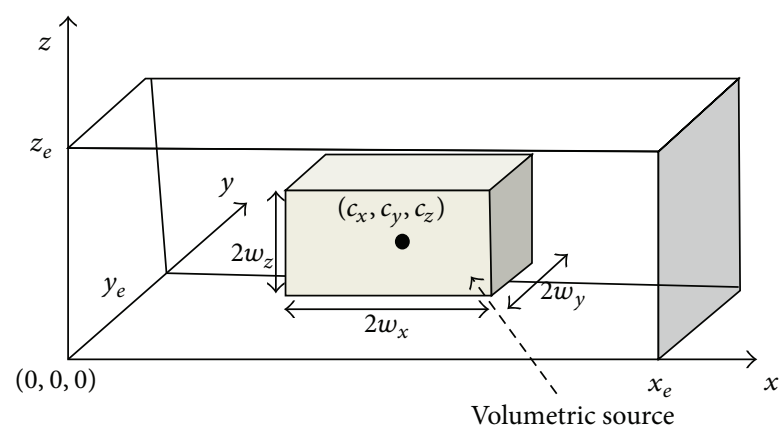

FIGURE 1: Schematic of volumetric source system.

This study is focused on the derivation and application of volumetric source model which represents pressure behavior or productivity is generalized for fractured reservoir; a new steady volumetric source model is applied to calculate pressure drop in hydraulic fractures for finite-conductivity assumption. Laplace transformation and orthogonal transformation are adopted to derive the solutions of two models. Productivity of fractured horizontal well is calculated by coupling the two models. An example of fractured horizontal well is studied; factors that influence the well productivity are analyzed.

\section{Volumetric Source Model}

2.1. Assumption. The porous media are assumed to be dualpermeability reservoir whose shape is a box with closed boundaries, and the size of the reservoir is $\left(x_{e}, y_{e}, z_{e}\right)$. A volumetric source with strength $q(t)$ produces oil in the reservoir, for which the central coordinate of the source is $\left(c_{x}, c_{y}, c_{z}\right)$, and the size is $\left(2 w_{x}, 2 w_{y}, 2 w_{z}\right)$. Figure 1 shows the schematic of the system.

2.2. Mathematical Model. In dual-porosity idealizations, Warren and Root introduced the dual-porosity model in terms of bulk properties. If $C_{\zeta}$ represents an intrinsic property of medium $\varsigma=m$ (matrix) or $f$ (natural fractures), then bulk property of medium $\varsigma$ is

$$
C_{\zeta, B}=C_{\varsigma} V_{\varsigma},
$$

where $V_{\varsigma}$ is the ratio of volume of medium $\varsigma, V_{m}+V_{f}=1$.

Characteristics of the matrix and natural fractures system are incorporated by the storativity $\omega$ and flow capacity ratio $\lambda$ is defined as follows:

$$
\omega=\frac{\left(\phi c_{t}\right)_{f, B}}{\left(\phi c_{t}\right)_{m, B}+\left(\phi c_{t}\right)_{f, B}}, \quad \lambda=\alpha \frac{k_{m, B}}{k_{f, B}} L^{2} .
$$

The pressure drop in the matrix or fracture system from initial pressure $p_{i}$ is

$$
P=p_{i}-p
$$

Dimensionless directions and time are

$$
l_{D}=\frac{l}{L}, \quad t_{D}=\frac{3.6 k_{f, B} t}{\mu L^{2}\left[\left(\phi c_{t}\right)_{m, B}+\left(\phi c_{t}\right)_{f, B}\right]} .
$$

Volumetric source model is

$$
\begin{gathered}
(1-\omega) \frac{\partial P_{m}}{\partial t_{D}}+\lambda\left(P_{m}-P_{f}\right)=0, \\
\frac{\partial^{2} P_{f}}{\partial x_{D}^{2}}+\frac{\partial^{2} P_{f}}{\partial y_{D}^{2}}+\frac{\partial^{2} P_{f}}{\partial z_{D}^{2}}+\lambda\left(P_{m}-P_{f}\right) \\
-\frac{B q\left(t_{D}\right) \mu}{3.6 k_{f, B} L V_{\text {source }, D}} h\left(x_{D}, y_{D}, z_{D}\right)=\omega \frac{\partial P_{f}}{\partial t_{D}}, \\
\left.P_{m}\right|_{t_{D}=0}=\left.P_{f}\right|_{t_{D}=0}=0, \\
\left.\frac{\partial P_{f}}{\partial l_{D}}\right|_{l_{D}=0}=0, \\
\left.\frac{\partial P_{f}}{\partial l_{D}}\right|_{l_{D}=l_{e D}}=0,
\end{gathered}
$$

where

$$
\begin{aligned}
h\left(x_{D}, y_{D}, z_{D}\right) \\
=\left[H\left(x_{D}-c_{x D}-w_{x D}\right)-H\left(x_{D}-c_{x D}+w_{x D}\right)\right] \\
\quad \times\left[H\left(y_{D}-c_{y D}-w_{y D}\right)-H\left(y_{D}-c_{y D}+w_{y D}\right)\right] \\
\quad \times\left[H\left(Z_{D}-c_{z D}-w_{z D}\right)-H\left(Z_{D}-c_{z D}+w_{z D}\right)\right] .
\end{aligned}
$$

$H\left(x-x_{0}\right)$ is Heaviside function:

$$
H\left(x-x_{0}\right)= \begin{cases}1 & x>x_{0} \\ 0 & x \leq x_{0}\end{cases}
$$

2.3. Solution of the Model. Take Laplace transformation in model (5):

$$
\bar{P}=L\left[P\left(t_{D}\right)\right]=\int_{0}^{\infty} P\left(t_{D}\right) e^{-s t_{D}} d t_{D}
$$

Volumetric source model after transformation is

$$
\begin{gathered}
\frac{\partial^{2} \bar{P}_{f}}{\partial x_{D}^{2}}+\frac{\partial^{2} \bar{P}_{f}}{\partial y_{D}^{2}}+\frac{\partial^{2} \bar{P}_{f}}{\partial z_{D}^{2}}-s f(s) \overline{P_{f}} \\
=\frac{B \bar{q} \mu}{3.6 k_{f, B} L V_{\text {source }, D}} h\left(x_{D}, y_{D}, z_{D}\right), \\
\left.\frac{\partial \bar{P}_{f}}{\partial l_{D}}\right|_{l_{D}=0}=0 \\
\left.\frac{\partial \bar{P}_{f}}{\partial l_{D}}\right|_{l_{D}=l_{e D}}=0
\end{gathered}
$$


where

$$
f(s)=\frac{\lambda+s \omega(1-\omega)}{(1-\omega) s+\lambda} .
$$

Corresponding to model (9), define the orthogonal transformation as

$$
\widetilde{P}=T\left(\bar{P}_{f}\right)=\int_{\Omega_{D}} E_{k, m, n}\left(x_{D}, y_{D}, z_{D}\right) \bar{P}_{f} d V_{D} .
$$

Inverse transformation formula is

$$
\bar{P}_{f}=\sum_{k=0}^{\infty} \sum_{m=0}^{\infty} \sum_{n=0}^{\infty} \frac{E_{k, m, n}}{\left\|E_{k, m, n}\right\|^{2}} \widetilde{P},
$$

where characteristic function of the orthogonal transformation is

$$
E_{k, m, n}\left(x_{D}, y_{D}, z_{D}\right)=\cos \frac{k \pi}{x_{e D}} x_{D} \cos \frac{m \pi}{y_{e D}} y_{D} \cos \frac{n \pi}{z_{e D}} z_{D},
$$

characteristic value is

$$
\gamma=\left(\frac{k \pi}{x_{e D}}\right)^{2}+\left(\frac{m \pi}{y_{e D}}\right)^{2}+\left(\frac{n \pi}{z_{e D}}\right)^{2},
$$

and norm of characteristic function is

$$
\begin{gathered}
\left\|E_{k, m, n}\left(x_{D}, y_{D}, z_{D}\right)\right\|^{2}=\frac{2^{\delta_{k k}+\delta_{m m}+\delta_{n n}}}{8} x_{e D} y_{e D} z_{e D}, \\
\delta_{k k}= \begin{cases}1 & k=0, \\
0 & k \neq 0 .\end{cases}
\end{gathered}
$$

After orthogonal transformation, the solution of the model is

$$
\widetilde{P}=-\frac{1}{\gamma+s f(s)} \frac{B \bar{q} \mu}{3.6 k_{f, B} L V_{\text {source }, D}} T\left[h\left(x_{D}, y_{D}, z_{D}\right)\right] .
$$

Applying the inverse transformation formula (12), volumetric source function in Laplace Space is

$$
\bar{P}_{f}=\bar{q} g_{\text {source }}\left(x_{D}, y_{D}, z_{D}\right),
$$

where $g_{\text {source }}\left(x_{D}, y_{D}, z_{D}\right)$ presents pressure drop at arbitrary point $\left(x_{D}, y_{D}, z_{D}\right)$ when volumetric source is created with unit strength

$$
\begin{gathered}
g_{\text {source }}\left(x_{D}, y_{D}, z_{D}\right)=\frac{B \mu}{3.6 k_{f, B} L V_{\text {source }, D}} \sum_{k, m, n=0}^{\infty} \frac{G_{\text {source }, k}\left(x_{D}\right) G_{\text {source }, m}\left(y_{D}\right) G_{\text {source }, m}\left(z_{D}\right)}{\gamma+s f(s)}, \\
G_{\text {source }, k}\left(l_{D}\right)= \begin{cases}\frac{2 w_{l D}}{l_{e D}} & k=0, \\
\frac{2 \cos \left(k \pi / l_{e D}\right) l_{D}\left[\sin \left(k \pi / l_{e D}\right)\left(c_{l D}+w_{l D}\right)-\sin \left(k \pi / l_{e D}\right)\left(c_{l D}-w_{l D}\right)\right]}{k \pi} & k \neq 0 .\end{cases}
\end{gathered}
$$

\section{Flow Model of Hydraulic Fracture}

3.1. Assumption of Flow in Hydraulic Fracture. The length of hydraulic fracture is $x_{F}$, height is $y_{F}$, and width is $w_{F}$. The flow in hydraulic fracture only occurs on the plane of the fracture. Oil from reservoir flows into the well through the hydraulic fracture immediately, and the pressure of hydraulic fracture recovers to reach balance at each time step.

Divide hydraulic fracture into $M \times N$ segments $(M$ segments in $x$ direction and $N$ segments in $y$ direction, both $M$ and $N$ are even which is shown in Figure 2). The $i$ th segment is described as $F_{\text {source }, i}$, in which central coordinate is $\left(x_{i}, y_{i}\right)$, total rate is $q_{F, i}$, and volume is $V_{i}\left(V_{i}=2 w_{F x, i} \times\right.$ $\left.2 w_{F y, i} \times w_{F}\right)$. The central coordinate of horizontal well which intersected by the hydraulic fracture is $\left(x_{w}, y_{w}\right)$, and the volume is $V_{w}\left(V_{w}=4 w^{2} \times w_{F}, w=\sqrt{\pi r_{w}^{2} / 4}\right)$.

3.2. Pressure Drop in Hydraulic Fracture. For each time step, pressure drop in hydraulic fracture is

$$
P_{F}(t)=p_{F, \text { ini }}(t)-p_{F},
$$

where $p_{F \text {,ini }}(t)$ is the average hydraulic fracture pressure at time $t$.

Assume that only the $i$ th segment $F_{\text {source }, i}$ flows to the well; flow model of hydraulic fracture is

$$
\begin{gathered}
\frac{\partial^{2} P_{F}}{\partial x^{2}}+\frac{\partial^{2} P_{F}}{\partial y^{2}}+\frac{B q_{F, i} \mu}{3.6 k_{F}}\left[\frac{h_{w}(x, y)}{V_{w}}-\frac{h_{i}(x, y)}{V_{i}}\right]=0, \\
\left.P_{F}\right|_{t=0}=0 \\
\left.\frac{\partial P_{F}}{\partial x}\right|_{x=0, x_{F}}=0 \\
\left.\frac{\partial P_{F}}{\partial y}\right|_{y=0, y_{F}}=0
\end{gathered}
$$

where

$$
\begin{aligned}
h_{i}(x, y)= & {\left[H\left(x-x_{i}-w_{F x, i}\right)-H\left(x-x_{i}+w_{F x, i}\right)\right] } \\
& \times\left[H\left(y-y_{i}-w_{F y, i}\right)-H\left(y-y_{i, 1}+w_{F y, i}\right)\right],
\end{aligned}
$$




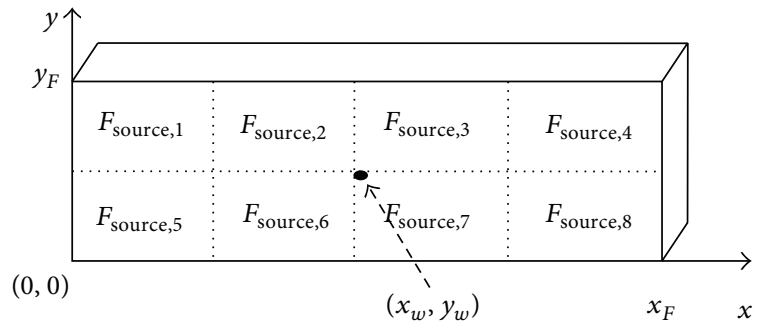

FIgURE 2: Cartesian coordinate system of hydraulic fractures (divided into $4 \times 2$ segments).

$$
\begin{aligned}
h_{w}(x, y)= & {\left[H\left(x-x_{w}-w_{w}\right)-H\left(x-x_{w}+w_{w}\right)\right] } \\
& \times\left[H\left(y-y_{w}-w_{w}\right)-H\left(y-y_{w}+w_{w}\right)\right] .
\end{aligned}
$$

Corresponding to model (20), the orthogonal transformation is defined as

$$
\widetilde{P}_{F}=T_{F}\left(P_{F}(x, y)\right)=\int_{0}^{x_{F}} \int_{0}^{y_{F}} P_{F} \cos \frac{k \pi}{x_{F}} x \cos \frac{m \pi}{y_{F}} y d x d y ;
$$

inverse transformation formula is

$$
P_{F}(x, y)=\sum_{k, m=1}^{\infty} \frac{F_{k, m}(x, y)}{\left\|F_{k, m}\right\|^{2}} \widetilde{P}_{F},
$$

where characteristic function of the orthogonal transformation is

$$
F_{k, m}(x, y)=\cos \frac{k \pi}{x_{F}} x \cos \frac{m \pi}{y_{F}} y
$$

characteristic value is

$$
\gamma_{F}=\left(\frac{k \pi}{x_{F}}\right)^{2}+\left(\frac{m \pi}{y_{F}}\right)^{2}
$$

and norm of characteristic function is

$$
\left\|F_{k, m}\right\|^{2}=\frac{x_{F} y_{F}}{4}
$$

Through application of the inverse transformation formula (23), pressure drop in hydraulic fracture is

$$
P_{F}(x, y)=\frac{B q_{F, i} \mu}{3.6 k_{F}} \sum_{k, m=1}^{\infty} \frac{F_{k, m}(x, y)}{\gamma_{F}\left\|F_{k, m}\right\|^{2}}\left(\frac{T_{k, w} T_{m, w}}{V_{w}}-\frac{T_{k, i} T_{m, i}}{V_{i}}\right),
$$

where

$$
\begin{aligned}
T_{k, i} & =\frac{x_{F}}{k \pi}\left[\sin \frac{k \pi}{x_{F}}\left(x_{i}+w_{F x, i}\right)-\sin \frac{k \pi}{x_{F}}\left(x_{i}-w_{F x, i}\right)\right], \\
T_{m, i}= & \frac{y_{F}}{m \pi}\left[\sin \frac{m \pi}{y_{F}}\left(y_{i}+w_{F y, i}\right)-\sin \frac{m \pi}{y_{F}}\left(y_{i}-w_{F y, i}\right)\right], \\
T_{k, w} & =\frac{x_{F}}{k \pi}\left[\sin \frac{k \pi}{x_{F}}\left(x_{w}+w_{w}\right)-\sin \frac{k \pi}{x_{F}}\left(x_{w}-w_{w}\right)\right], \\
T_{m, i} & =\frac{y_{F}}{m \pi}\left[\sin \frac{m \pi}{y_{F}}\left(y_{w}+w_{w}\right)-\sin \frac{m \pi}{y_{F}}\left(y_{w}-w_{w}\right)\right] .
\end{aligned}
$$

Pressure difference from arbitrary point of hydraulic fracture to the well is

$$
\Delta P_{F}(x, y)=q_{F, i} R_{i}(x, y),
$$

where

$$
\begin{array}{r}
R_{i}(x, y) \\
=\frac{B \mu}{3.6 k_{F}} \sum_{k, m=1}^{\infty} \frac{\left[F_{k, m}\left(x_{w}, y_{w}\right)-F_{k, m}(x, y)\right]}{\gamma_{F}\left\|F_{k, m}\right\|^{2}} \\
\cdot\left(\frac{T_{k, w} T_{m, w}}{V_{w}}-\frac{T_{k, i} T_{m, i}}{V_{i}}\right) .
\end{array}
$$

According to the superposition theory, when all the segments of hydraulic fracture flow to the well, pressure difference from arbitrary point to the well is

$$
\Delta P_{F}(x, y)=\sum_{i=1}^{N \times M} q_{F, i} R_{i}(x, y) .
$$

Take Laplace transformation to (31); the pressure difference in Laplace Space is

$$
\Delta \bar{P}_{F}(x, y)=\sum_{i=1}^{N \times M} \bar{q}_{F, i} R_{i}(x, y) .
$$

\section{Productivity of Fractured Horizontal Well}

The $j$ th hydraulic fracture is divided into $(N \times M)^{(j)}$ segments, and the size is $\left(w_{F}^{(j)}, x_{F}^{(j)}, y_{F}^{(j)}\right)$. Then $N_{t}\left(N_{t}=\sum_{j=1}^{N_{H}}(N \times\right.$ $M)^{(j)}$ ) volumetric source exists in the reservoir, matrix of each source's rate in Laplace Space is $\overline{\mathbf{q}}=\left(\bar{q}_{1}, \bar{q}_{2}, \ldots, \bar{q}_{N_{t}}\right)$, $3 \mathrm{D}$ coordinate is $\left(\mathbf{r}_{1}, \mathbf{r}_{2}, \ldots, \mathbf{r}_{N_{t}}\right)$, and $2 \mathrm{D}$ coordinate is $\left(\mathbf{r}_{F, 1}, \mathbf{r}_{F, 2}, \ldots, \mathbf{r}_{F, N_{t}}\right)$.

According to the pressure drop caused by flowing in reservoir and hydraulic fracture, the problem can be written in the matrix formation as

$$
\left(\mathbf{A}_{1}+\mathbf{A}_{2}\right) \overline{\mathbf{q}}=\overline{\mathbf{b}},
$$


TABLE 1: Parameters of an example.

\begin{tabular}{lccc}
\hline$x_{e}(\mathrm{~m})$ & 500 & $N_{H}$ & 5 \\
$y_{e}(\mathrm{~m})$ & 500 & $x_{F}(\mathrm{~m})$ & 200 \\
$z_{e}(\mathrm{~m})$ & 20 & $y_{F}(\mathrm{~m})$ & 10 \\
$L_{H}(\mathrm{~m})$ & 500 & $w_{F}(\mathrm{~m})$ & 0.003 \\
$r_{w}(\mathrm{~m})$ & 0.05 & $k_{F}\left(\mu \mathrm{m}^{2}\right)$ & 50 \\
$k_{m}\left(\mu \mathrm{m}^{2}\right)$ & 0.005 & $p_{i}(\mathrm{MPa})$ & 20 \\
$\phi_{m}$ & 0.15 & $p_{w}(\mathrm{MPa})$ & 10 \\
$k_{f}\left(\mu \mathrm{m}^{2}\right)$ & 1 & $\mu(\mathrm{mPa} \cdot \mathrm{s})$ & 2 \\
$V_{f}$ & 0.005 & $c_{t}(1 / \mathrm{MPa})$ & 0.0005 \\
$\lambda$ & 3000 & $B$ & 1.08 \\
$\omega$ & 0.02 & $t$ day $)$ & 100 \\
\hline
\end{tabular}

where

$$
\begin{aligned}
& \overline{\mathbf{b}}=\left(\bar{P}_{w}, \bar{P}_{w}, \ldots, \bar{P}_{w}\right)^{T}, \\
& \mathbf{A}_{\mathbf{1}}=\left(a_{i j}^{(1)}\right)_{N_{t} \times N_{t}}, \quad a_{i j}^{(1)}=g_{j}\left(\mathbf{r}_{i}\right) \\
& \mathbf{A}_{2}=\left(\begin{array}{llll}
\mathbf{A}_{2,1} & & & \\
& \mathbf{A}_{2,2} & & \\
& & \ddots & \\
& & & \mathbf{A}_{2, \mathrm{~N}_{\mathrm{H}}}
\end{array}\right) \text {, } \\
& \mathbf{A}_{\mathbf{2}, \mathbf{k}}=\left(a_{i, j}^{2, k}\right)_{(N \times M)^{(k)} \times(N \times M)^{(k)}} \\
& a_{i, j}^{2, k}=R_{J}\left(\mathbf{r}_{F I}\right), \\
& I= \begin{cases}i & k=1, \\
\sum_{l=1}^{k-1}(N \times M)^{(l)}+i & k>1,\end{cases} \\
& J= \begin{cases}j & k=1, \\
\sum_{l=1}^{k-1}(N \times M)^{(l)}+j & k>1 .\end{cases}
\end{aligned}
$$

The matrix of each source's rate $\overline{\mathbf{q}}$ can be calculated, and the total flow rate of the well in Laplace Space $\bar{Q}$ is

$$
\overline{\mathrm{Q}}=\sum_{j=1}^{N_{t}} \bar{q}_{j} .
$$

According to Stehfest algorithm, the flow rate of the well in real time space can be calculated numerically.

\section{Influential Factors Analysis}

Volumetric source function in this paper is generalized from the case in homogeneous reservoirs developed by Valkó and Amini [11], and validation of volumetric source function has also been tested. In the paper, the volumetric source model will be verified by analytical flow model [6]. Table 1 shows the basic parameter values of this simulation.

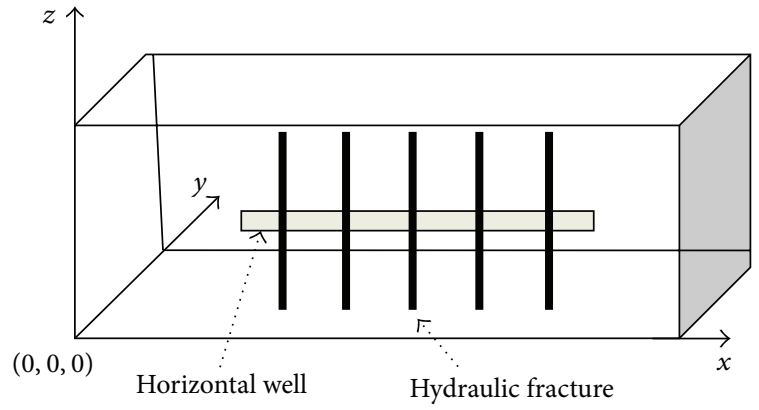

FIgURE 3: Schematic of fractured horizontal well.

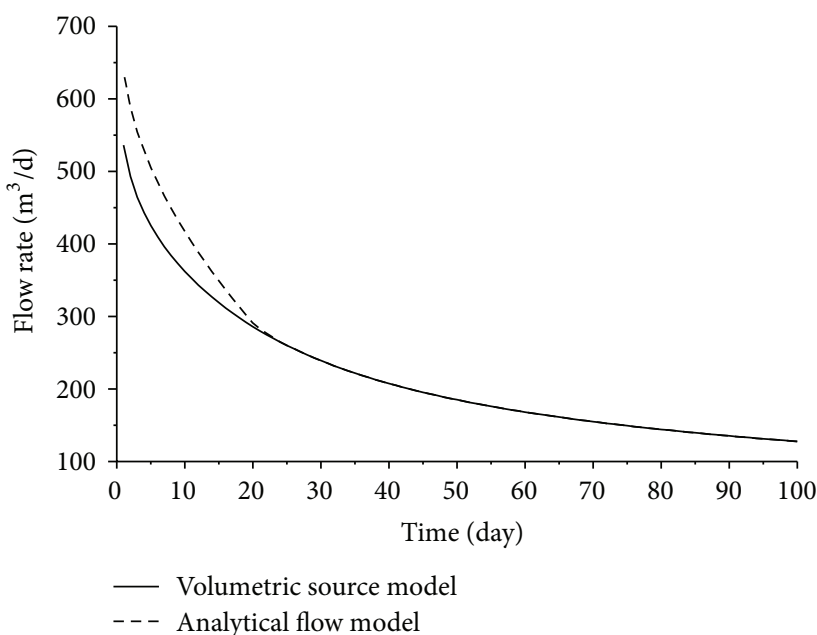

Figure 4: Flow rate verses time.

Figure 3 shows the schematic of the simulated example, five evenly spaced hydraulic fractures are distributed along the horizontal well, and each hydraulic fracture is divided into volumetric sources. Production-time relationship simulated by two methods is shown in Figure 4, which shows great consistency except for the very beginning of production. As the analytical flow model was proposed based on linear flow assumption, the flow resistance is underestimated at the beginning of production, which leads to result of analytical flow model which is higher than the result of volumetric source models.

Figure 5 implies that the contribution of each hydraulic fracture is different. Hydraulic fractures at the end of horizontal well dominate large portion of reservoir and have less interference with other fractures. Therefore, they have greater contribution than fractures in middle part. Ratio of flow rate shown in Figure 5 is defined as

$$
\text { Ratio of flow rate }=\frac{\text { flow rate of hydraulic fracture }}{\text { total flow rate }} .
$$

5.1. Factors of Hydraulic Fractures. In this subsection, influences of factors of hydraulic fractures on productivity are analyzed. In order to reveal the effects of hydraulic fracture 


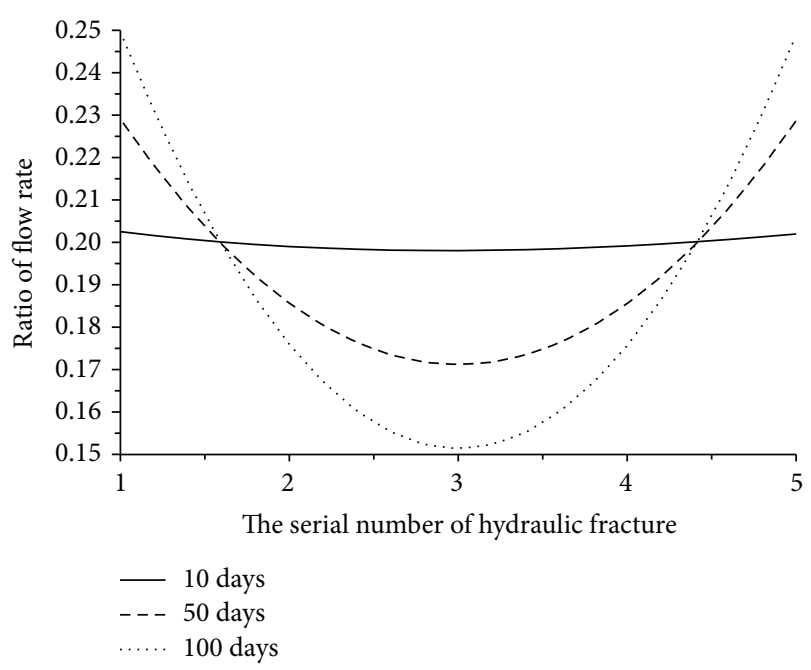

FIGURE 5: Distribution of ratio of flow rate.

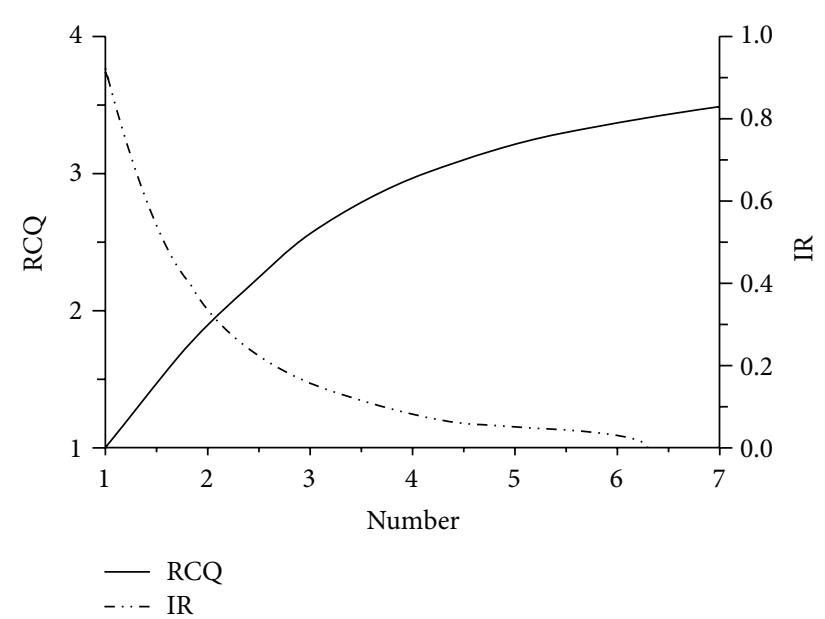

FIGURE 6: Ratio of cumulative production and increment rate for different number.

and reservoir factors, incremental rate (IR) and ratio of cumulative production (RCQ) are adopted:

$$
\begin{gathered}
\operatorname{IR}(x)=\frac{\mathrm{CQ}(x+1)-\mathrm{CQ}(x)}{\operatorname{CQ}(x)}, \\
\operatorname{RCQ}=\frac{\operatorname{CQ}(x)}{\operatorname{CQ}\left(x_{0}\right)},
\end{gathered}
$$

where $x$ represents factor value, such as value of number or length or conductivity; $x_{0}$ represents the minimal factor's value simulated; CQ represents cumulative production of horizontal well.

With increasing number, the flow rate increases rapidly at the beginning, but interference between hydraulic fractures shows up earlier, and flow rate decreases faster with time. Figure 6 presents the ratios of cumulative production and increment rate for different number of hydraulic fractures. As illustrated, increment rate is less than 0.1 as the number of hydraulic fracture exceeds 4-5; cumulative production is

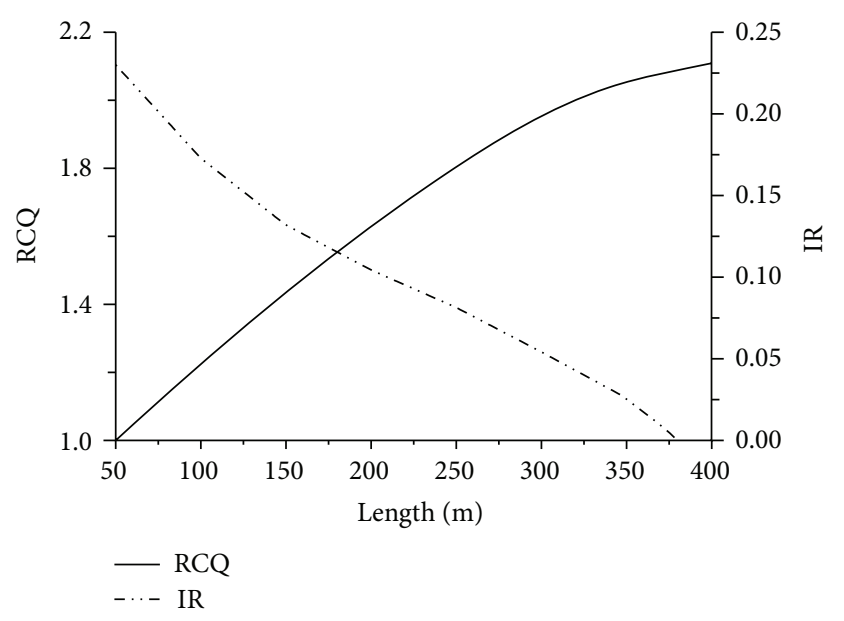

FIGURE 7: Ratio of cumulative production and increment rate for different length.

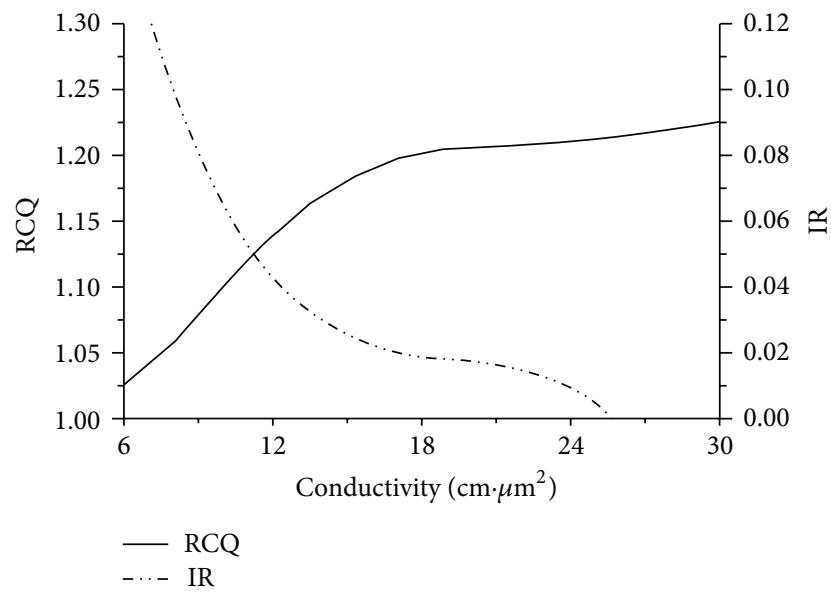

FIGURE 8: Ratio of cumulative production and increment rate for different conductivity.

more than 3 times larger than that of a single hydraulic fracture.

Figure 7 reveals effect of length on productivity of horizontal well. One of the major advantages of hydraulic fracturing is increasing the surface area in contact reservoir. For a certain number, it is accomplished by increasing the length of hydraulic fracture. Result shows that increment rate is less than 0.1 if length of hydraulic fracture approaches $250 \mathrm{~m}$.

Another mechanism for hydraulic fracturing to improve productivity of horizontal well is creating high-conductivity flow path. In this case, conductivity should remain at $20 \mathrm{~cm} \cdot \mu \mathrm{m}^{2}-30 \mathrm{~cm} \cdot \mu \mathrm{m}^{2}$ (see Figure 8).

Comparing the results of Figures $6-8$, it is obvious that the increasing number improves productivity most significantly, while increasing conductivity improves the least. In unconventional reservoirs, flow capacity of reservoirs may be not sufficient to supply the hydraulic fractures to its full flow capacity. Therefore, higher conductivity of hydraulic fractures is undesirable once basic flow capacity of reservoir is achieved. 


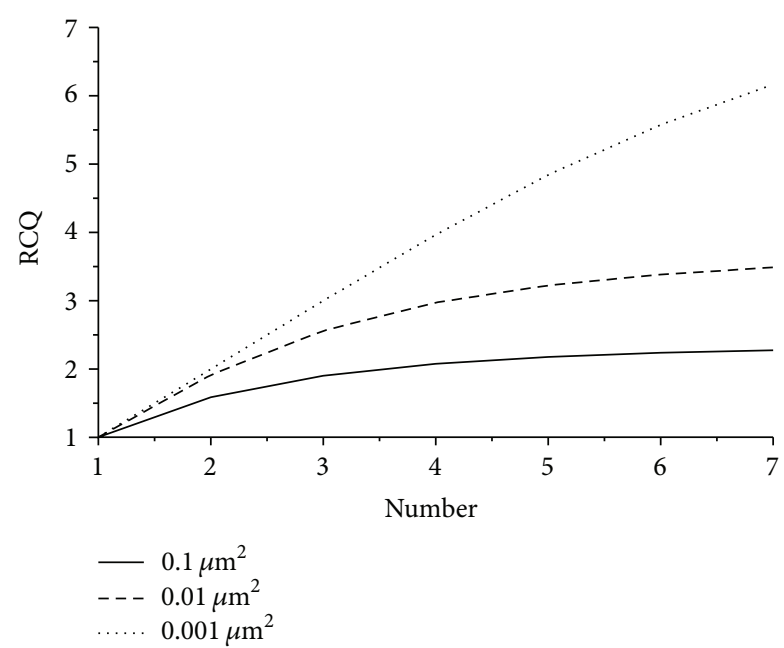

FIGURE 9: Effect of number on ratios of cumulative production under different permeability.

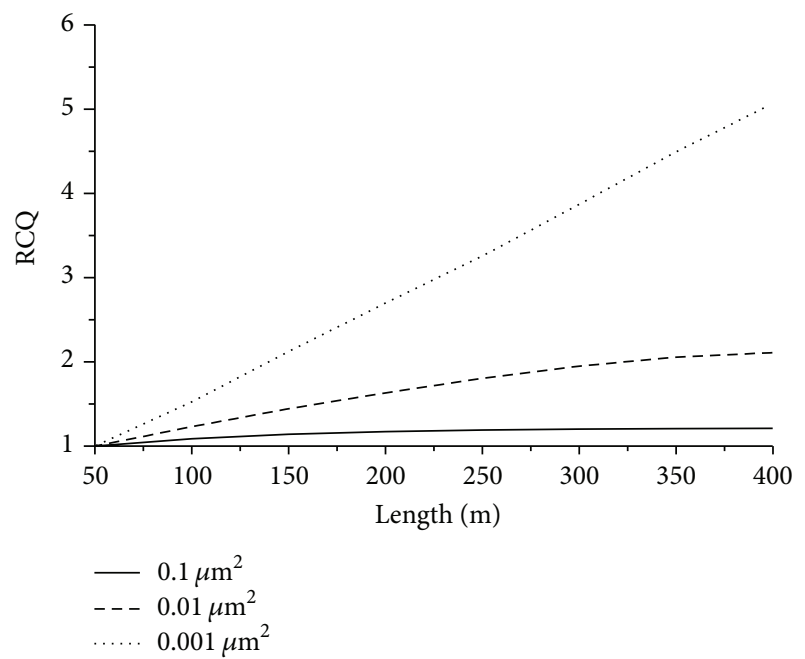

FIGURE 10: Effect of length on ratios of cumulative production under different permeability.

The simulated example reveals a clear method to design hydraulic fracturing by applying volumetric source model. For a real horizontal well, parameters of hydraulic fractures could be optimally designed by following the method.

5.2. Permeability of Reservoir. Flow capacity of reservoir is the primary factor that affects designing of hydraulic fracturing, which is controlled by natural fractures in the dual-porosity idealization. Figures 9-11 show the effects of hydraulic fractures on ratios of cumulative production when permeability of reservoir is $0.001 \mu \mathrm{m}^{2}-0.1 \mu \mathrm{m}^{2}$. When permeability of reservoir reduces the range of low-permeability, oil flows with more difficulty, and interference between hydraulic fractures will show up much later. Therefore, increasing the surface area in contacted reservoir seems more important, and the high-conductivity is less vital for hydraulic fractures, which can easily satisfy the flow capacity of reservoir. This outcome

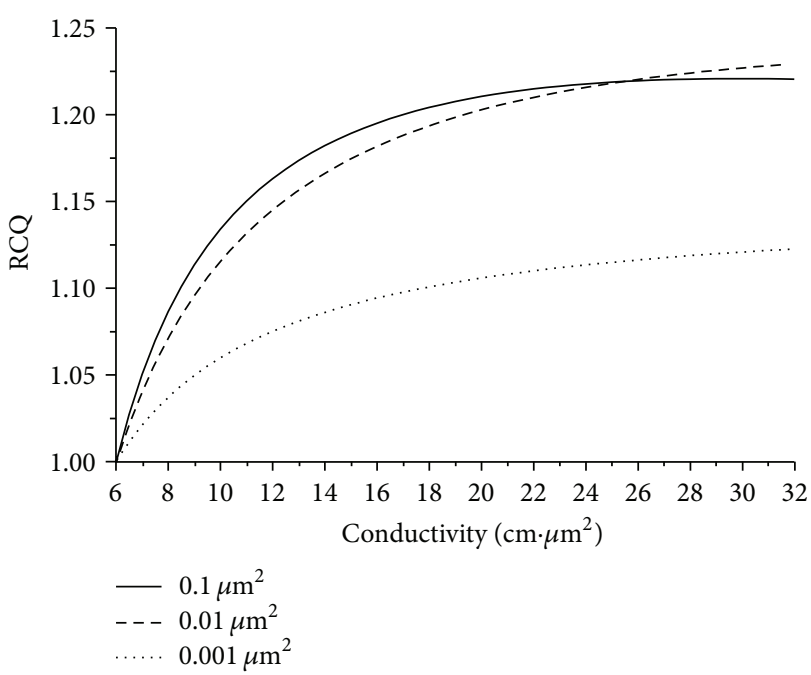

FIGURE 11: Effect of conductivity on ratios of cumulative production under different permeability.

emphasizes the importance of improving contacted area when designing hydraulic fracturing.

\section{Conclusions}

(1) New volumetric source models of flow in reservoir and hydraulic fractures are developed to simulate the productivity of fractured horizontal well in naturally fractured reservoir, which is verified by analytical flow model.

(2) Compared with traditional point source functions, the volumetric source function brings many advantages. Firstly, it shows no inherent singularity, and pressure can be calculated at any point in reservoir. Secondly, it is more adjustable; the solution is always in the same form, regardless of the types of the source. Last, it is more convenient for programming calculation.

(3) A practical example is simulated to show the essence of productivity of fractured horizontal wells. Result shows that flow rate of each fracture is different and the inner fracture contributes least to productivity. Increasing number improves productivity most significantly, while increasing length is the second important factor, and increasing conductivity is the least.

(4) The defined parameters IR and RCQ could be employed to optimize number, length, and conductivity of hydraulic fractures. More and longer hydraulic fractures are required for lower permeability reservoirs, but larger conductivity is unnecessary.

\section{Nomenclature}

$x_{e}(\mathrm{~m})$ : Length of reservoir

$y_{e}(\mathrm{~m})$ : Width of reservoir

$z_{e}(\mathrm{~m})$ : Height of reservoir 


$\begin{array}{ll}c_{x}(\mathrm{~m}): & \begin{array}{l}\text { Position of the center of the source } \\ \text { in } x \text { direction }\end{array} \\ c_{y}(\mathrm{~m}): & \begin{array}{l}\text { Position of the center of the source } \\ \text { in } y \text { direction }\end{array} \\ c_{z}(\mathrm{~m}): & \text { Position of the center of the source } \\ & \text { in } z \text { direction } \\ w_{x}(\mathrm{~m}): & \text { Source width in } x \text { direction } \\ w_{y}(\mathrm{~m}): & \text { Source width in } y \text { direction } \\ w_{z}(\mathrm{~m}): & \text { Source width in } z \text { direction } \\ q\left(\mathrm{~m}^{3} / \mathrm{d}\right): & \text { Strength of the source } \\ V(\mathrm{dimensionless}): & \text { The ratio of volume } \\ \alpha\left(\mathrm{m}^{-2}\right): & \text { Shape factor } \\ k(\mathrm{D}): & \text { Permeability } \\ \left(\phi c_{t}\right)\left(\mathrm{MPa}{ }^{-1}\right): & \text { Storage } \\ \mu(\mathrm{mPa} \cdot \mathrm{s}): & \text { Viscosity } \\ L(\mathrm{~m}): & \text { Reference length } \\ p_{i}(\mathrm{MPa}): & \text { Initial pressure } \\ p(\mathrm{MPa}): & \text { Pressure } \\ \lambda(\mathrm{dimensionless}): & \text { Flow capacity ratio } \\ \omega(\text { dimensionless }): & \text { Storativity. }\end{array}$

\section{Subscript}

$m$ : Matrix

$f$ : Natural fractures

D: Dimensionless

$B$ : Bulk property.

\section{Conflict of Interests}

The authors declare that there is no conflict of interests regarding the publication of this paper.

\section{Acknowledgment}

This study is supported by the National Science and Technology Major Project of China (Grant no. 2011ZX05022-006004HZ).

\section{References}

[1] M. Brown, E. Ozkan, R. Raghavan, and H. Kazemi, "Practical solutions for pressure transient responses of fractured horizontal wells in unconventional reservoirs," in Proceedings of the SPE Annual Technical Conference and Exhibition, New Orleans, La, USA, 2009, SPE 125043.

[2] M. Brown, E. Ozkan, R. Raghavan, and H. Kazemi, "Comparison of fractured-horizontal-well performance in tight sand and shale reservoirs," SPE Reservoir Evaluation \& Engineering, vol. 14, no. 2, pp. 248-259, 2014.

[3] A. Hasan, A triple-porosity model for fractured horizontal wells [M.S. thesis], Texas A\&M University, 2010.

[4] X. Wang, W. Luo, X. Hou, and J. Wang, "Transient pressure analysis of multiple-fractured horizontal wells in boxed reservoirs," Petroleum Exploration and Development, vol. 41, no. 1, pp. 74-78, 2014.

[5] L. I. Longlong, Y. Jun, Y. Li, M. Wu, Q. Zeng, and R. Lu, "Productivity calculation and distribution of staged multi-cluster fractured horizontal wells," Petroleum Exploration and Development, vol. 41, no. 4, pp. 504-508, 2014.

[6] L. Haitao, W. Junchao, W. Yongqing, J. Beibei, and L. Wei, “Generalized productivity model for designing hydraulic fractures in horizontal wells located in naturally fissured low-permeability gas reservoirs," Chemistry and Technology of Fuels and Oils, vol. 50, no. 4, pp. 299-314, 2014.

[7] R. Raghavan and C. Chih-Cheng, "An analysis of horizontal wells intercepted by multiple fractors," SPE Journal, vol. 2, no. 3, pp. 235-245, 1997.

[8] C.-C. Chen and R. Raghavan, "A multiply-fractured horizontal well in a rectangular drainage region," SPE Journal, vol. 2, no. 4, pp. 455-462, 1997.

[9] A. Zerzar and Y. Bettam, "Interpretation of multiple hydraulically fractured horizontal well in closed systems," in Proceedings of the Canadian International Petroleum Conference, PETSOC2004-027, June 2004.

[10] J. Guo, F. Zeng, J. Zhao, and Y. Xu, "A new model to predict fractured horizontal well production," in Proceedings of the Canadian International Petroleum Conference, Calgary, Canada, June 2006.

[11] P. P. Valkó and S. Amini, "The method of distributed volumetric sources for calculating the transient and pseudosteadystate productivity of complex well-fracture configurations," in Proceedings of the Hydraulic Fracturing Technology Conference, SPE 106279, pp. 426-439, January 2007.

[12] D. Zhu, F. Magalhaes, and P. P. Valko, "Predicting productivity of multiple-fractured horizontal gas wells," in Proceedings of the SPE Hydraulic Fracturing Technology Conference, SPE 106280, College Station, Tex, USA, January 2007.

[13] F. Magalhaes, D. Zhu, S. Amini, and P. P. Valko, "Optimization of fractured-well performance of horizontal gas wells," in Proceedings of the SPE 2nd International Oil Conference and Exhibition, SPE 108779, pp. 503-509, Veracruz, Mexico, June 2007.

[14] S. Amini and P. P. Valkó, "Using distributed volumetric sources to predict production from multiple-fractured horizontal wells under non-darcy-flow conditions," SPE Journal, vol. 15, no. 1, pp. 105-115, 2010.

[15] P. Q. Lian, L. S. Cheng, and J. Y. Cui, "A new computation model of fractured horizontal well coupling with reservoir," International Journal for Numerical Methods in Fluids, vol. 67, no. 8, pp. 1047-1056, 2011.

[16] J. Lin and D. Zhu, "Predicting well performance in complex fracture systems by slab source method," in Proceedings of the Hydraulic Fracturing Technology Conference, SPE 151960, The Woodlands, Tex, USA, February 2012.

[17] S. K. Siddiqui, A. Ali, and H. Dehghanpour, "New advances in production data analysis of hydraulically fractured tight reservoirs," in Proceedings of the SPE Canadian Unconventional Resources Conference, SPE 162830, Calgary, Canada, OctoberNovember 2012.

[18] A. Alkouh, D. Schechter, R. A. Wattenbarger et al., "Practical use of simulators for characterization of shale reservoirs," in Proceedings of the SPE Canadian Unconventional Resources Conference, SPE-162645-MS, October-November 2012.

[19] E. Ozkan and R. Raghavan, "New solutions for well-test-analysis problems. Part 1. Analytical considerations," SPE Formation Evaluation, vol. 6, no. 3, pp. 359-368, 1991.

[20] E. Ozkan and R. Raghavan, "New solutions for well-test-analysis problems. Part 2. Computational considerations and applications," SPE Formation Evaluation, vol. 6, no. 3, pp. 369-378, 1991. 
[21] E. Ozkan and R. Raghavan, "New solutions for well-test-analysis problems: part III-additional algorithms," in Proceedings of the Annual Technical Conference and Exhibition, SPE 28424, New Orleans, La, USA, September 1994.

[22] A. C. Gringarten and H. J. Ramey Jr., "The use of source and Green's functions in solving unsteady-flow problems in reservoir," Society of Petroleum Engineers Journal, vol. 13, no. 5, pp. 285-296, 1973.

[23] B. O. Ogunsanya, T. P. Oetama, J. F. Lea, L. R. Heinze, and P. S. Adisoemarta, "A coupled model for analyzing transient pressure behavior of horizontal drain-holes," in Proceedings of the SPE Production and Operations Symposium, pp. 619-632, April 2005.

[24] B. O. Ogunsanya, T. P. Oetama, J. F. Lea, L. R. Heinze, and P. S. Adisoemarta, "A robust type curve solution for analyzing pressure-transient behaviors of both vertical and horizontal fractures systems," in Proceedings of the Annual SPE International Technical Conference and Exhibition, SPE 105979, Abuja, Nigeria, August 2006. 


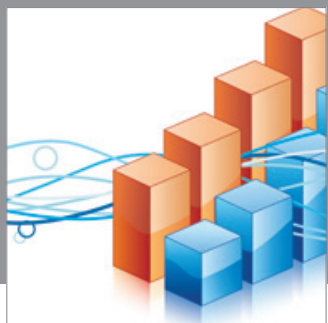

Advances in

Operations Research

mansans

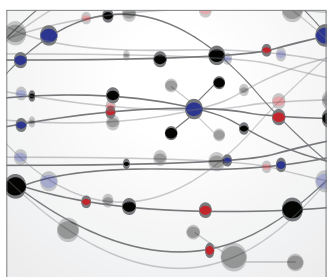

The Scientific World Journal
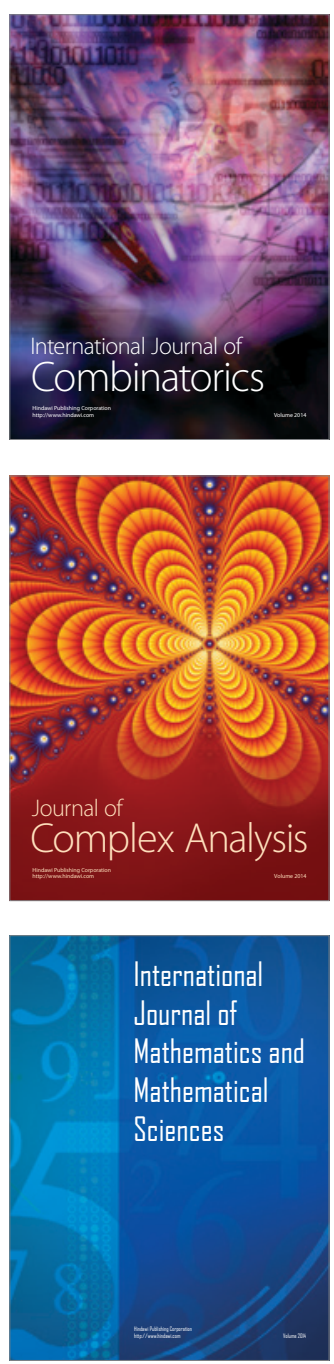
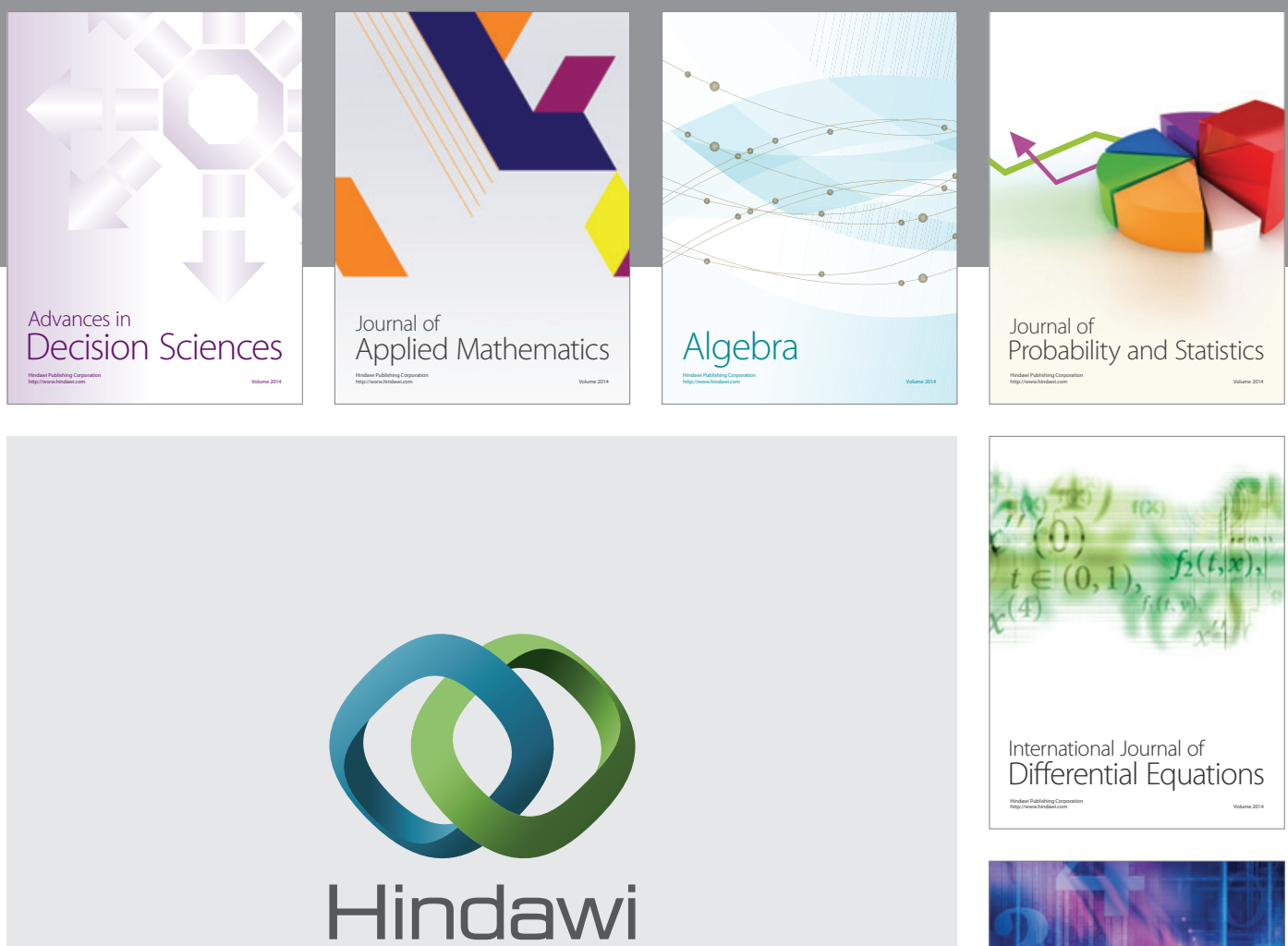

Submit your manuscripts at http://www.hindawi.com
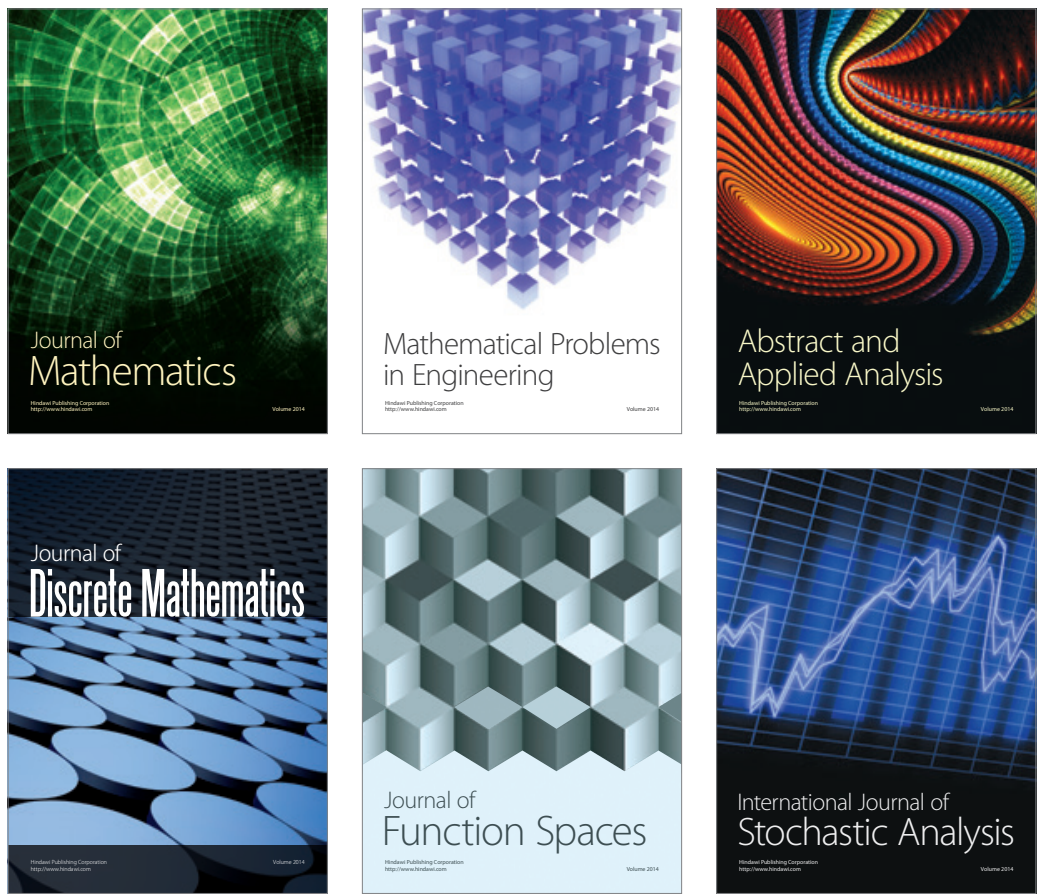

Journal of

Function Spaces

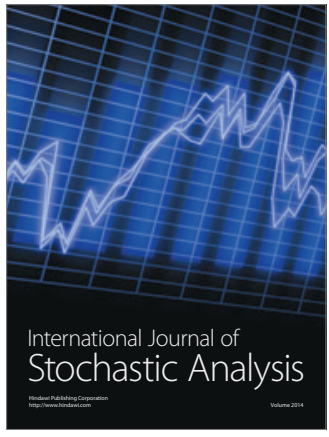

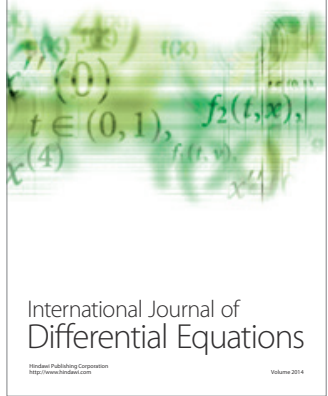
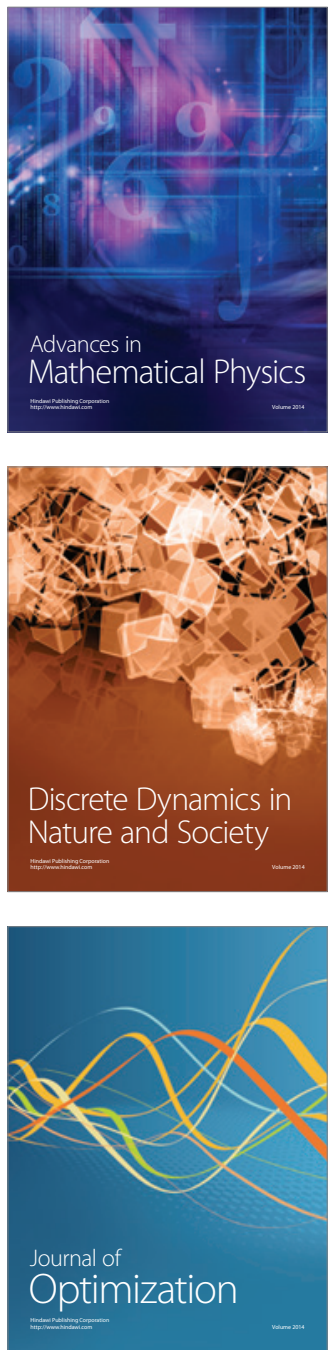